Wright State University

CORE Scholar

$10-1-2007$

\title{
Introduction and Recovery of Ga and N Sublattice Defects in Electron-Irradiated Gan
}

\author{
F. Tuomisto \\ V. Ranki \\ David C. Look \\ Wright State University - Main Campus, david.look@wright.edu \\ Gary C. Farlow \\ Wright State University - Main Campus, gary.farlow@wright.edu
}

Follow this and additional works at: https://corescholar.libraries.wright.edu/physics

Part of the Physics Commons

\section{Repository Citation}

Tuomisto, F., Ranki, V., Look, D. C., \& Farlow, G. C. (2007). Introduction and Recovery of Ga and N Sublattice Defects in Electron-Irradiated Gan. Physical Review B, 76 (16), 165207.

https://corescholar.libraries.wright.edu/physics/182

This Article is brought to you for free and open access by the Physics at CORE Scholar. It has been accepted for inclusion in Physics Faculty Publications by an authorized administrator of CORE Scholar. For more information, please contact library-corescholar@wright.edu. 


\title{
Introduction and recovery of Ga and $\mathrm{N}$ sublattice defects in electron-irradiated GaN
}

\author{
F. Tuomisto* and V. Ranki \\ Laboratory of Physics, Helsinki University of Technology, P.O. Box 1100, 02015 TKK, Finland \\ D. C. Look \\ Semiconductor Research Center, Wright State University, Dayton, Ohio 45435, USA \\ and Materials and Manufacturing Directorate, Air Force Research Laboratory, Wright-Patterson AFB, Ohio 45433, USA \\ G. C. Farlow \\ Physics Department, Wright State University, Dayton, Ohio 45435, USA
}

(Received 31 May 2007; revised manuscript received 7 September 2007; published 24 October 2007)

\begin{abstract}
We have used positron annihilation spectroscopy to study the introduction and recovery of point defects introduced by 0.45 and $2 \mathrm{MeV}$ electron irradiation at room temperature in $n$-type GaN. Isochronal annealings were performed up to $1220 \mathrm{~K}$. We observe vacancy defects with specific lifetime of $\tau_{V}=190 \pm 15$ ps that we tentatively identify as $\mathrm{N}$ vacancies or related complexes in the neutral charge state in the samples irradiated with $0.45 \mathrm{MeV}$ electrons. The $\mathrm{N}$ vacancies are produced at a rate $\Sigma_{\mathrm{N}}^{0.45} \simeq 0.25 \mathrm{~cm}^{-1}$. The irradiation with $2 \mathrm{MeV}$ electrons produces negatively charged $\mathrm{Ga}$ vacancies and negative nonopen volume defects (negative ions) originating from the Ga sublattice, at a rate $\Sigma_{\mathrm{Ga}}^{2.0} \simeq 5 \mathrm{~cm}^{-1}$. The irradiation-induced $\mathrm{N}$ vacancies anneal out of the samples at around $600 \mathrm{~K}$, possibly due to the motion of the irradiation-induced N interstitials. Half of the irradiation-induced $\mathrm{Ga}$ vacancies anneal out of the samples also around $600 \mathrm{~K}$, and this is interpreted as the isolated $\mathrm{Ga}$ vacancies becoming mobile with a migration barrier of $E_{M}^{V, \mathrm{Ga}}=1.8 \pm 0.1 \mathrm{eV}$. Interestingly, we observe a change of charge state of the irradiation-induced negative ions from 2- to 1- likely due to a reconstruction of the defects in two stages at annealing temperatures of about 600 and $700 \mathrm{~K}$. The negative ions anneal out of the samples together with the other half of the Ga vacancies (stabilized by, e.g., N vacancies and/or hydrogen) in thermal annealings at $800-1100 \mathrm{~K}$.
\end{abstract}

DOI: 10.1103/PhysRevB.76.165207

PACS number(s): 61.72.Ji, 61.82.Fk, 71.55.Eq, 78.70.Bj

\section{INTRODUCTION}

Gallium nitride and its alloys form a class of important semiconductor materials for applications in optoelectronics at blue and ultraviolet wavelengths and electronic devices operating at high temperatures and high voltage. The importance of intrinsic point defects in $\mathrm{GaN}$ is due to their role in determining the electrical and optical properties of the material, as well as their influence on the material behavior in various processing steps such as ion implantation and thermal annealing. Point defects are created in semiconductor materials during growth, where their formation is governed by thermodynamics and growth kinetics. They can be introduced in concentrations much larger than those given by the thermodynamic equilibrium by means of irradiation of the material, e.g., by electrons. The study of the formation of point defects under these nonequilibrium conditions and in the subsequent annealing treatments yield important information on the basic physical properties of the semiconductor material.

The defects introduced in electron irradiation of $\mathrm{GaN}$ have been studied earlier with electrical and optical methods, ${ }^{1-3}$ optical detection of electron paramagnetic resonance (ODEPR), ${ }^{4-7}$ and positron annihilation spectroscopy ${ }^{8}$ as well. However, only in the most recent studies ${ }^{2,3,7}$ could the measurements be performed in samples with both low impurity content and low dislocation densities, ${ }^{9-11}$ allowing more accurate observations of the isolated intrinsic point defects. In most of these studies, the irradiations were performed with electrons of $2 \mathrm{MeV}$ (or higher) energy, in which case damage is created in both $\mathrm{Ga}$ and $\mathrm{N}$ sublattices. Recent experiments $^{2,3}$ show that $\mathrm{N}$ sublattice defects could be selectively introduced by using $0.42 \mathrm{MeV}$ electrons.

The ODEPR experiments, ${ }^{4-7}$ performed after irradiations at cryogenic temperatures, have shown that the isolated positively charged $\mathrm{Ga}$ interstitials are mobile already below room temperature. However, they may be complexed with other irradiation-induced defects or residual impurities and survive thermal treatments up to $800 \mathrm{~K} .^{4,5}$ The more recent ODEPR results suggest that the isolated negatively charged Ga vacancies could also be stable up to $800 \mathrm{~K},{ }^{7}$ while the earlier positron experiments performed after room-temperature $2 \mathrm{MeV}$ electron irradiation indicate that the isolated $\mathrm{Ga}$ vacancies (produced at a rate of $1 \mathrm{~cm}^{-1}$ ) anneal out of $\mathrm{GaN}$ samples already at $600 \mathrm{~K}^{8}{ }^{8}$ The results of the irradiations performed with low-energy $(0.42 \mathrm{MeV})$ electrons ${ }^{2}$ suggest that $\mathrm{N}$ vacancy donors are produced at a rate of $0.02 \mathrm{~cm}^{-1}$ and $\mathrm{N}$ interstitial acceptors at a lower rate of $0.01 \mathrm{~cm}^{-1}$.

In this work, we investigate the introduction and thermal recovery of the point defects created in GaN by roomtemperature irradiation with 0.45 and $2 \mathrm{MeV}$ electrons. To do this, we apply positron annihilation spectroscopy, which is sensitive to defects with open volume. Positrons are trapped at neutral and negative vacancy defects due to the missing positive ion core. The reduced electron density at vacancies increases the positron lifetime. In addition to vacancies, at low temperatures, positrons can be trapped at the hydrogenic states around negatively charged nonopen volume defects. In this case, the positron lifetime is the same as 
in the host lattice due to the wide radius of the hydrogenic state.

We observe vacancy defects with specific lifetime of $\tau_{V}$ $=190 \pm 15$ ps that we tentatively identify as $\mathrm{N}$ vacancies or related complexes in the neutral charge state in the samples irradiated with $0.45 \mathrm{MeV}$ electrons. The $\mathrm{N}$ vacancies are produced at a rate $\Sigma_{\mathrm{N}}^{0.45} \simeq 0.25 \mathrm{~cm}^{-1}$. The irradiation with $2 \mathrm{MeV}$ electrons produces negatively charged $\mathrm{Ga}$ vacancies and negative nonopen volume defects (negative ions) at similar concentrations, at a rate $\Sigma_{\mathrm{Ga}}^{2.0} \simeq 5 \mathrm{~cm}^{-1}$. The irradiation-induced $\mathrm{N}$ vacancies anneal out of the samples at around $600 \mathrm{~K}$, possibly due to the motion of the irradiationinduced $\mathrm{N}$ interstitials. Half of the irradiation-induced $\mathrm{Ga}$ vacancies anneal out of the samples also around $600 \mathrm{~K}$, and this is interpreted as the isolated $\mathrm{Ga}$ vacancies becoming mobile with a migration barrier of $E_{M}^{V, \mathrm{Ga}}=1.8 \pm 0.1 \mathrm{eV}$. Interestingly, we observe a change of charge state of the irradiationinduced negative ions from $2-$ to $1-$ due to a reconstruction of the defects in two stages at annealing temperatures of about 600 and $700 \mathrm{~K}$. The negative ions anneal out of the samples together with the other half of the Ga vacancies (stabilized by, e.g., $\mathrm{N}$ vacancies and/or hydrogen) in thermal annealings at $800-1100 \mathrm{~K}$.

\section{METHOD}

\section{A. Experimental details}

The positron lifetime experiments were performed in undoped high-purity freestanding GaN crystals grown by hydride vapor phase epitaxy (HVPE). Two samples were irradiated with $2 \mathrm{MeV}$ electrons and two other with $0.45 \mathrm{MeV}$ electrons, to fluences of $5 \times 10^{17}$ and $2 \times 10^{17} \mathrm{~cm}^{-2}$, respectively. The irradiations were performed with a current density of $2 \mu \mathrm{A} / \mathrm{cm}^{2}$ at room temperature, in a vacuum of 2 $\times 10^{-6}$ torr. The as-grown samples were $n$-type: $N_{e} \simeq 5$ $\times 10^{15} \mathrm{~cm}^{-3}$ due to residual $\mathrm{O}\left([\mathrm{O}] \simeq 10^{16} \mathrm{~cm}^{-3}\right)$. The samples become highly resistive after the $2 \mathrm{MeV}$ electron irradiation, while the conductivity does not change significantly in the $0.45 \mathrm{MeV}$ electron irradiation.

After the $0.45 \mathrm{MeV}$ electron irradiation, the samples were mounted to a closed-cycle liquid helium cryostat for positron measurements as a function of isochronal annealing between 300 and $600 \mathrm{~K}$. The 30 min annealings were performed in situ in the positron measurement cryostat in a vacuum of $10^{-6} \mathrm{mbar}$. The positron lifetime measurements were performed at $300 \mathrm{~K}$, and a measurement temperature scan from 20 to $300 \mathrm{~K}$ was performed after selected annealing steps. The samples irradiated with $2 \mathrm{MeV}$ electrons were mounted to a positron measurement setup equipped with liquid nitrogen cooling, where $30 \mathrm{~min}$ in situ annealings could be performed up to a temperature of $600 \mathrm{~K}$. The samples were further annealed in a vacuum (about $10^{-3} \mathrm{mbar}$ ) furnace up to $1220 \mathrm{~K}$. The Gibbs free energy at this high temperature and low pressure is, although being at the limit, still lower for GaN than for its constituents. ${ }^{12}$ The positron lifetime measurements were performed at $300 \mathrm{~K}$, and measurement temperature scans from 80 to $500 \mathrm{~K}$ were performed after selected annealing steps.
The positron lifetimes were measured with a conventional fast-fast coincidence spectrometer with a time resolution of 250 ps. ${ }^{13}$ Two identical sample pieces were sandwiched with a $20 \mu \mathrm{Ci}$ positron source $\left({ }^{22} \mathrm{Na}\right.$ deposited on $1.5 \mu \mathrm{m}$ Al foil). Typically, $2 \times 10^{6}$ annihilation events were collected in each positron lifetime spectrum in the annealing experiments, with the exception of the spectra measured for the separation of lifetime components in the $0.45 \mathrm{MeV}$ electron-irradiated samples, where the number was $2 \times 10^{7}$. The lifetime spectrum $n(t)=\Sigma_{i} I_{i} \exp \left(-t / \tau_{i}\right)$ was analyzed as the sum of exponential decay components convoluted with the Gaussian resolution function of the spectrometer, after subtracting the constant background and annihilations in the source material (215 ps, 2.9\%; 400 ps, 3.5\%; 1500 ps, $0.25 \%$ ). The positron in state $i$ annihilates with a lifetime $\tau_{i}$ and an intensity $I_{i}$. The state in question can be the delocalized state in the lattice or the localized state at a vacancy defect. The increase of the average lifetime $\tau_{\text {ave }}=\Sigma_{i} I_{i} \tau_{i}$ above the bulk lattice lifetime $\tau_{B}$ shows that vacancy defects are present in the material. This parameter is insensitive to the decomposition procedure and even as small a change as $1 \mathrm{ps}$ in its value can be reliably measured.

\section{B. Data analysis}

\section{Positron trapping at defects}

The temperature dependence of the average positron lifetime is analyzed with the model of trapping and escape rates of positrons, explained in detail in earlier works. ${ }^{13-16}$ In this model, the trapping coefficient $\mu_{V}$ to a neutral vacancy is independent of temperature and to a negatively charged vacancy it varies as $T^{-0.5}$. The trapping rate of positrons into the vacancies (concentration $c_{V}$ ) is $\kappa_{V}=\mu_{V} c_{V}$. Positrons can also get trapped at hydrogenlike Rydberg states surrounding negative-ion-type defects (shallow traps for positrons). The positron trapping rate at the Rydberg state $\mu_{R}$ varies also as $T^{-0.5}$, which is the result predicted by theory for the transition from a free state to a bound state in a Coulomb potential. ${ }^{16}$ The thermal escape rate from the Rydberg state can be written as ${ }^{13}$

$$
\delta_{\mathrm{st}}=\mu_{R}\left(\frac{m_{+} k_{B} T}{2 \pi \hbar^{2}}\right)^{3 / 2} \exp \left(-E_{b, \mathrm{st}} / k_{B} T\right)
$$

where $\mu_{R}$ is the positron trapping coefficient to the lowest hydrogenlike Rydberg state, $E_{b, \mathrm{st}}$ is the positron binding energy of the lowest Rydberg state (typically $<0.1 \mathrm{eV}$ ), and $m_{+} \simeq m_{0}$ is the effective mass of the positron. As the binding energy is proportional to the square of the charge state of the defect, $Z^{2}$, different binding energies in the same material (with the same dielectric constant) reflect defects with different charge states. In principle, positrons can also escape from the Rydberg states around negatively charged vacancies, but we assume that the transition from the Rydberg state to the ground state in the vacancy is fast enough so that this effect can be neglected. This is supported by the results obtained previously. ${ }^{17}$ An effective trapping rate of the shallow traps can thus be defined as ${ }^{13}$ 


$$
\kappa_{\mathrm{st}}^{\mathrm{eff}}=\frac{\kappa_{\mathrm{st}}}{1+\delta_{\mathrm{st}} / \lambda_{\mathrm{st}}},
$$

where $\lambda_{\mathrm{st}} \simeq \lambda_{B}$ is the annihilation rate of positrons trapped at the Rydberg state, which coincides with the annihilation rate $\lambda_{B}$ from the delocalized state in the bulk lattice and $\kappa_{\mathrm{st}}$ $=\mu_{R} c_{\text {st }}$ is directly related to the concentration of the negative ions.

\section{Kinetic trapping model}

The decomposition of the lifetime spectra into several lifetime components gives the possibility to determine experimentally the fractions of positrons annihilating in various states. The average lifetime can be written as ${ }^{13}$

$$
\tau_{\mathrm{ave}}=\eta_{B} \tau_{B}+\sum_{j} \eta_{D, j} \tau_{D, j}
$$

where $\eta_{B}$ and $\tau_{B}$ are the annihilation fraction and positron lifetime in the free state in the lattice, and $\eta_{D, j}$ and $\tau_{D, j}$ are the corresponding values in bound states at the defect $D_{j}$. The annihilation fractions are related to the trapping rates through $^{13}$

$$
\eta_{B}=\frac{\lambda_{B}}{\lambda_{B}+\sum_{j} \kappa_{D, j}^{\mathrm{eff}}}, \quad \eta_{D, j}=\frac{\kappa_{D, j}^{\mathrm{eff}}}{\lambda_{B}+\sum_{j^{\prime}} \kappa_{D, j^{\prime}}^{\mathrm{eff}}} .
$$

Equation (3) can be fitted to the $\tau_{\text {ave }}$ vs $T$ data using the trapping rates and the possible binding energies to the Rydberg states as fitting parameters. Another possibility is to write [solving Eqs. (3) and (4)] the trapping rate $\kappa_{D, j}^{\text {eff }}$ to a defect $D_{j}$ as a function of the experimental parameters:

$$
\kappa_{D, j}^{\mathrm{eff}}=\lambda_{B} \frac{\tau_{\mathrm{ave}}-\tau_{B}}{\tau_{D, j}-\tau_{\mathrm{ave}}}-\sum_{j^{\prime} \neq j} \kappa_{D, j^{\prime}}^{\text {eff }} \frac{\tau_{D, j^{\prime}}-\tau_{\mathrm{ave}}}{\tau_{D, j}-\tau_{\mathrm{ave}}} .
$$

In the case of only one type of defect, the sum in Eq. (5) vanishes and the trapping rate can be calculated directly from the experimental values of $\tau_{\mathrm{ave}}, \tau_{D, j}$, and $\tau_{B}$. If several defect types are present, the trapping rates to the other defects need to be known.

\section{RESULTS}

The results of the positron lifetime measurements in the as-grown and irradiated ( 0.45 and $2 \mathrm{MeV}$ electrons) $\mathrm{GaN}$ samples are presented in Fig. 1. At 300-500 K, the average positron lifetime in the as-grown sample is constant and provides the lifetime of the positron in the delocalized state in the GaN lattice, $\tau_{B}=161 \mathrm{ps}$ at $300 \mathrm{~K}$, in good agreement with previous experiments in defect-free GaN. ${ }^{18,19}$ The increase in the average positron lifetime with decreasing temperature at $80-300 \mathrm{~K}$ is a clear indication of the presence of negatively charged vacancies, the positron trapping coefficient of which increases with decreasing temperature. The vacancy concentration of these vacancies is close to the detection limit of the method and thus these vacancies are not observed at room temperature. The lifetime spectra could be decomposed into two lifetime components below $150 \mathrm{~K}$,

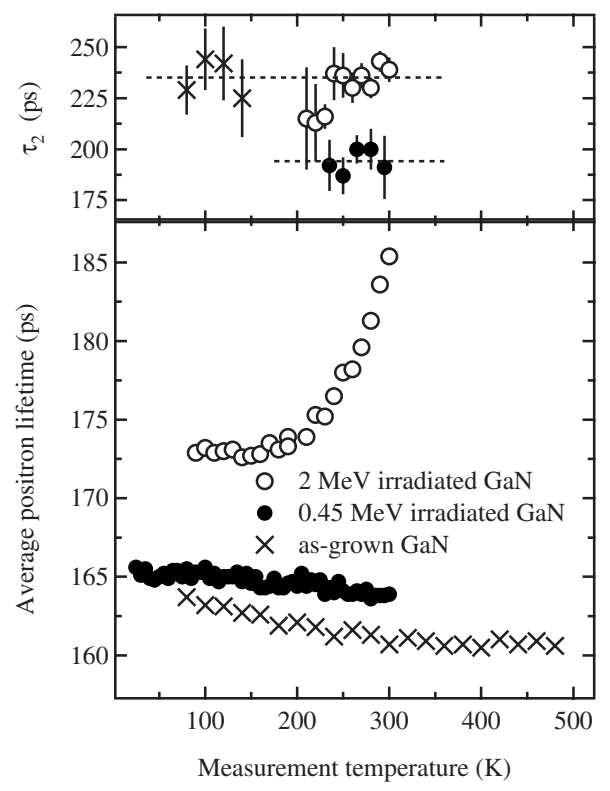

FIG. 1. The average positron lifetime in the as-grown and irradiated GaN samples as a function of measurement temperature. The corresponding second (higher) lifetime components separated from the spectra are shown in the upper panel.

giving $\tau_{2}=235 \pm 15 \mathrm{ps}$ for the higher component. This lifetime component can be attributed to the Ga vacancy, which is in the negative charge state and most likely complexed with oxygen. The concentration of the Ga vacancies in the as-grown $\mathrm{GaN}$ samples can be estimated from the average lifetime as $\left[V_{\mathrm{Ga}}\right] \simeq 4 \times 10^{15} \mathrm{~cm}^{-3}$, in good agreement with the previously observed relation between $\mathrm{O}$ impurities and Ga vacancies in HVPE GaN. ${ }^{20}$ No other negatively charged defects are observed in the as-grown samples, indicating that their concentrations are below $10^{15} \mathrm{~cm}^{-3}$.

After irradiation, the average positron lifetime is higher than in the as-grown reference (Fig. 1), indicating that vacancy defects are produced in the irradiations with both electron energies. The average positron lifetime is constant or slightly increasing with decreasing temperature in the $0.45 \mathrm{MeV}$ electron-irradiated samples. This indicates that the vacancy defects introduced in the irradiation are in the neutral charge state. On the other hand, the constant behavior of the average positron lifetime at $80-180 \mathrm{~K}$ and the subsequent strong increase at $200-300 \mathrm{~K}$ in the $2 \mathrm{MeV}$ electronirradiated samples is a clear indication of both negatively charged vacancies and negative-ion-type defects being produced in the irradiation. For both irradiated states, the lifetime spectra could be decomposed into two lifetime components at temperatures above $200 \mathrm{~K}$, where the effect of the irradiation-induced vacancies is the largest in the $0.45 \mathrm{MeV}$ electron-irradiated samples and the effect of the negativeion-type defects is the smallest in the $2 \mathrm{MeV}$ electronirradiated samples. The higher component in the $2 \mathrm{MeV}$ electron-irradiated samples is $\tau_{2}=235 \pm 10 \mathrm{ps}$, and it can be attributed to $\mathrm{Ga}$ vacancies as in the case of as-grown samples. Interestingly, the higher lifetime component in the $0.45 \mathrm{MeV}$ electron-irradiated samples is clearly lower, $\tau_{2}$ $=190 \pm 15 \mathrm{ps}$, indicating that the size of the observed va- 


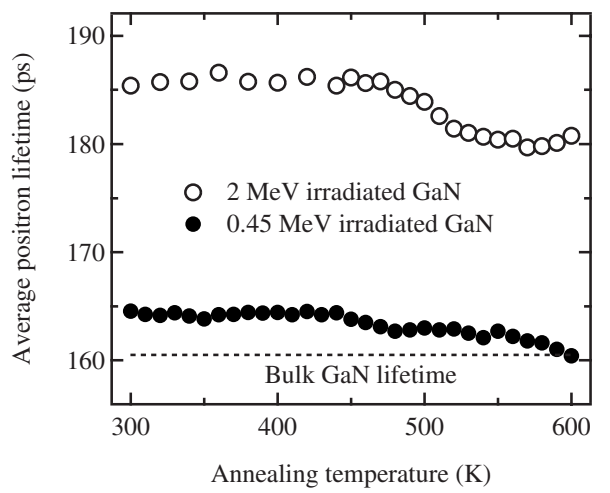

FIG. 2. The average positron lifetime measured at room temperature in the irradiated $\mathrm{GaN}$ samples as a function of the annealing temperature.

cancy defect is significantly smaller than that of the Ga vacancy. This suggests that positrons annihilate at irradiationinduced $\mathrm{N}$ vacancies in the neutral charge state.

Figure 2 shows the evolution of the average positron lifetime measured at room temperature in the irradiated $\mathrm{GaN}$ samples with the annealing temperature. The average lifetime starts decreasing in both 0.45 and $2 \mathrm{MeV}$ electronirradiated samples after the annealing at $450 \mathrm{~K}$. The average lifetime measured in the $2 \mathrm{MeV}$ electron-irradiated samples stops decreasing at about $550 \mathrm{~K}$, staying at a level $20 \mathrm{ps}$ higher than that in the as-grown samples. This indicates that a part of the irradiation-induced Ga vacancies anneals out in the annealing at $450-550 \mathrm{~K}$, but a significant concentration remains in the material. On the other hand, the average lifetime measured in the $0.45 \mathrm{MeV}$ electron-irradiated samples reaches the as-grown level after the annealing at $600 \mathrm{~K}$, above which no further evolution is observed. This indicates that all the irradiation-induced defects have annealed out of the material after the annealing at $600 \mathrm{~K}$. As can be seen in Fig. 3, the average positron lifetime coincides with that measured in the as-grown samples. This recovery temperature is the same as that assigned to the migration of $\mathrm{N}$ vacancies in previous electrical measurements of irradiated $\mathrm{GaN} .{ }^{1}$

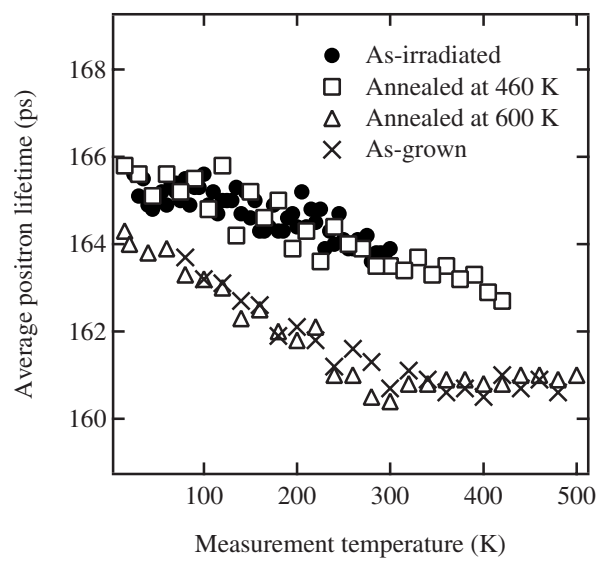

FIG. 3. The average positron lifetime measured as a function of temperature in the $0.45 \mathrm{MeV}$ electron-irradiated $\mathrm{GaN}$ samples after selected annealing temperatures.

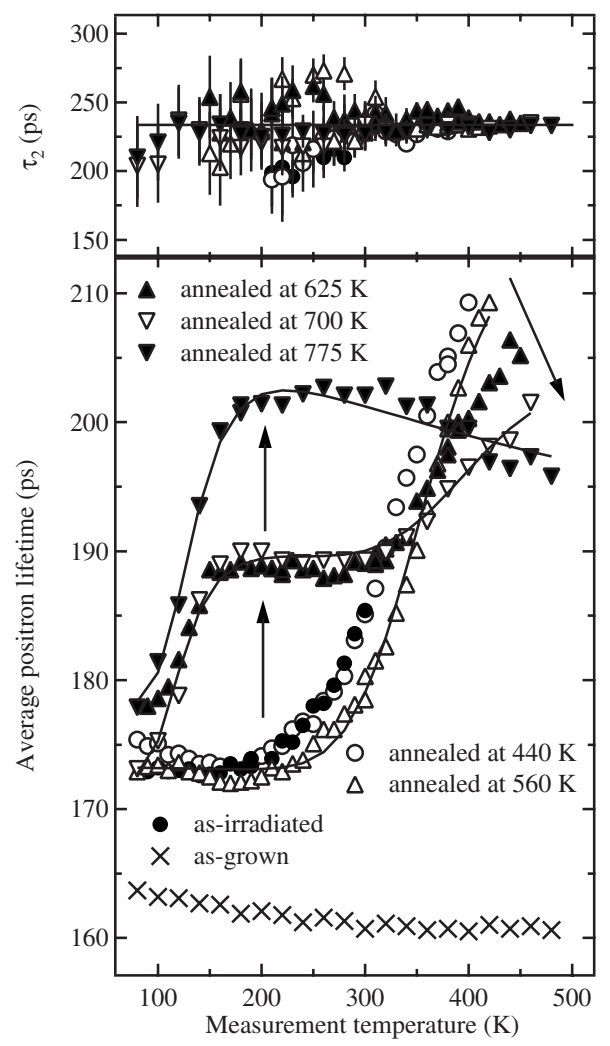

FIG. 4. The average positron lifetime measured as a function of temperature in the $2 \mathrm{MeV}$ electron-irradiated GaN samples after selected annealing temperatures up to $775 \mathrm{~K}$. The arrows indicate the direction of increasing annealing temperature. The solid lines are fits to the data using Eqs. (1)-(5). The corresponding second (higher) lifetime components separated from the spectra are shown in the upper panel.

As only a part of the irradiation-induced defects anneals out at $600 \mathrm{~K}$ from the $2 \mathrm{MeV}$ electron-irradiated $\mathrm{GaN}$ samples, annealings at higher temperatures were performed in a vacuum furnace. Figures 4 and 5 show the average positron lifetime measured as function of temperature after selected annealing steps in the range $440-1220 \mathrm{~K}$. The higher lifetime component could be separated from the lifetime spectra measured between the annealings, shown in the upper panels of the figures. Its value remains constant $\left(\tau_{2}\right.$ $=235 \pm 15 \mathrm{ps}$ ) throughout the whole experiment, indicating that the Ga vacancies are the dominant open volume defect observed in the samples. Small dips in the second lifetime component can be observed where the effect of the negative ions is the strongest due to the mixing of the lifetime components in the separation procedure.

Figure 4 shows the average positron lifetime after selected annealing steps in the range $440-775 \mathrm{~K}$. The data show that no changes occur up to the annealing temperature of $440 \mathrm{~K}$. Above this annealing temperature, the average lifetime measured at $200-400 \mathrm{~K}$ starts decreasing, indicating that Ga vacancies start annealing out from the material, as seen in the data measured after the annealing at $560 \mathrm{~K}$. After the annealing at $625 \mathrm{~K}$, a significant change in the data is observed. The average lifetime measured at $150-300 \mathrm{~K}$ increases by 


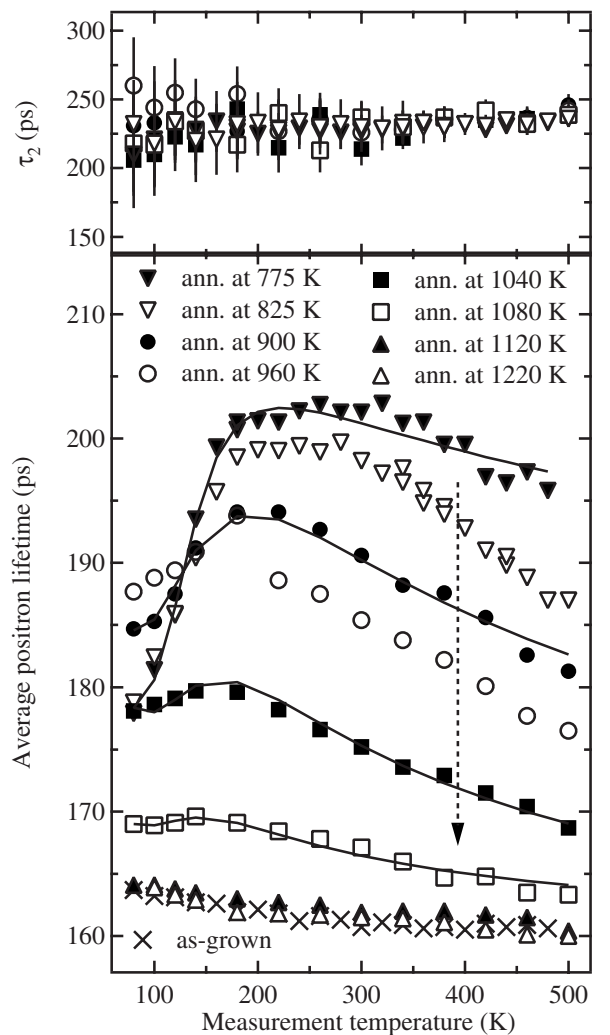

FIG. 5. The average positron lifetime measured as a function of temperature in the $2 \mathrm{MeV}$ electron-irradiated $\mathrm{GaN}$ samples after selected annealing temperatures from 775 to $1220 \mathrm{~K}$. The arrow indicates the direction of increasing annealing temperature. The solid lines are fits to the data. The corresponding second (higher) lifetime components separated from the spectra measured in the samples annealed at most at $1080 \mathrm{~K}$ are shown in the upper panel.

$15 \mathrm{ps}$, accompanied by a further decrease at measurement temperatures above $300 \mathrm{~K}$. In addition, the average lifetime measured at $80-100 \mathrm{~K}$ remains unchanged. The behavior of the average lifetime as a function of temperature in the range $300-450 \mathrm{~K}$ indicates that a part (but not all) of the irradiation-induced negative-ion-type defects have recovered after the annealing at $625 \mathrm{~K}$. Interestingly, the presence of another negative-ion-type defect with a lower positron binding energy is evident from the behavior at $80-150 \mathrm{~K}$. This issue is discussed in more detail in Sec. IV. No significant changes are observed in the data after the annealings at $650-700 \mathrm{~K}$. At annealing temperatures $700-775 \mathrm{~K}$, the average lifetime measured at $150-300 \mathrm{~K}$ increases by another $15 \mathrm{ps}$, indicating that the rest of the negative-ion-type defects active close to room temperature anneal out. No significant changes in the Ga vacancy or low binding energy negative ion concentrations are observed.

Figure 5 shows the average positron lifetime measured as a function of temperature after selected annealing steps in the range $775-1220 \mathrm{~K}$. The data show that the negative-ion-type defects and the $\mathrm{Ga}$ vacancies that remain in the material gradually anneal out from the samples, and all the irradiation-induced defects have recovered after the annealing at $1120 \mathrm{~K}$. No further evolution up to an annealing temperature of $1220 \mathrm{~K}$ was observed.

\section{DISCUSSION}

\section{A. Defects introduced in the irradiation}

\section{1. $0.45 \mathrm{MeV}$ electron irradiation}

We suggest that the lifetime $\tau_{2}=190 \pm 15$ ps observed after the irradiation with $0.45 \mathrm{MeV}$ electrons is due to the nitrogen vacancy. The Doppler broadening spectrum (not shown) measured in the samples does not change in the irradiation when compared to the bulk. This provides support for the identification of the defect as the $\mathrm{N}$ vacancy, as the changes are expected to be minimal due to the similar overlap with the $\mathrm{Ga} 3 d$ electrons both in the GaN lattice and in the $\mathrm{N}$ vacancy. Also, the results of theoretical calculations for an outward relaxed $\mathrm{N}$ vacancy suggest that the lifetime could be 20-30 ps above the bulk lifetime. ${ }^{21}$ In addition, Ga vacancies filled with even two hydrogen atoms, that could be expected to result in a similar lifetime component, would still give a clear signal in the Doppler broadening, ${ }^{20}$ while Ga vacancies complexed with more than two hydrogen atoms are unlikely to occur in $n$-type material. ${ }^{22}$ Based on the fact that the average positron lifetime in Figs. 1 and 3 is almost constant as function of temperature, the observed $\mathrm{N}$ vacancy is in the neutral charge state. The slight decrease of the average lifetime with increasing temperature can be attributed to initial negatively charged Ga vacancies present in the material already before the irradiation.

Assuming a positron trapping coefficient of $\mu_{V, \mathrm{~N}} \simeq 1$ $\times 10^{15} \mathrm{~s}^{-1}$, typical of small neutral vacancies, we estimate the $\mathrm{N}$ vacancy concentration from the value of $\tau_{\mathrm{ave}}=164 \mathrm{ps}$ at $300 \mathrm{~K}$ as $\left[V_{\mathrm{N}}\right] \simeq 5 \times 10^{16} \mathrm{~cm}^{-3}$. The irradiation fluence was $\phi(0.45 \mathrm{MeV})=2 \times 10^{17} \mathrm{~cm}^{-2}$, which gives an introduction rate $\Sigma_{N}^{0.45}=\left[V_{N}\right] / \phi \simeq 0.25 \mathrm{~cm}^{-1}$. No Ga vacancy production is observed in the irradiation with $0.45 \mathrm{MeV}$ electrons, in good agreement with the minimum electron energy of $0.53 \mathrm{MeV}$ needed for the $\mathrm{Ga}$ displacement predicted by theory. $^{2}$

The introduction rate of $70 \mathrm{meV}$ donors in $0.42 \mathrm{MeV}$ electron irradiation has been observed to be only $0.02 \mathrm{~cm}^{-1}$. $^{2}$ These donors were attributed to the isolated $\mathrm{N}$ vacancies. On the other hand, taking our estimate of the positron trapping coefficient to be of the correct order of magnitude, we observe neutral $\mathrm{N}$ vacancies with a production rate an order of magnitude higher than that of the $\mathrm{N}$ vacancies acting as donors. We propose that the $\mathrm{N}$ vacancies observed with positrons have formed neutral complexes with hydrogen, present at high enough concentrations in the samples, a possibility suggested by theory. ${ }^{23}$ Hence, they would not contribute to the electrical measurements. Acceptor-type defects were also observed to be introduced in the irradiation in Ref. 2, but with half the introduction rate of the donors. Our data are in good agreement with this observation, as such low a concentration of additional negatively charged defects would not be observed due to the higher concentrations of both irradiationinduced neutral $\mathrm{N}$ vacancies and the in-grown negatively charged $\mathrm{Ga}$ vacancies.

\section{2. $2 \mathrm{MeV}$ electron irradiation}

The $2 \mathrm{MeV}$ electron irradiation produces $\mathrm{Ga}$ vacancies with the positron lifetime of $\tau_{2}=235 \pm 10 \mathrm{ps}$. The conven- 
tional $S$ and $W$ (the Doppler broadening data are presented in Ref. 20) parameters obtained from the Doppler broadening spectra measured in the samples fall on the line connecting the bulk and Ga vacancy-specific points, confirming the identification. In addition to the Ga vacancies, negative-iontype defects (likely Ga interstitials and/or antisites) are also produced, causing the decrease of the average positron lifetime with decreasing temperature, as seen in Figs. 1, 4, and 5.

The concentration of the irradiation-induced Ga vacancies can be estimated from the average positron lifetime measured at $400 \mathrm{~K}$ after the annealing at $440 \mathrm{~K}$, which has not caused any changes in the data measured at $300 \mathrm{~K}$ or below. The Ga vacancies are in the negative charge state, which is demonstrated by the constant average lifetime below $200 \mathrm{~K}$ : if the $\mathrm{Ga}$ vacancies were in the neutral charge state, the average lifetime would continue decreasing toward the bulk lifetime. The data indicate that some of the positrons annihilate as trapped at the negative ions even at $400 \mathrm{~K}$. Hence, in order to estimate the Ga vacancy concentration, we add 5 ps to the average lifetime measured at $400 \mathrm{~K}: \tau_{\mathrm{ave}}^{*}=\tau_{\text {ave }}+5 \mathrm{ps}$ (the slope of the $\tau_{\text {ave }}$ vs $T$ becomes less steep when approaching $400 \mathrm{~K}$ ). The uncertainty inherent in this adjustment is at most a factor of 1.5. Assuming a positron trapping coefficient of $\mu_{V, \mathrm{Ga}} \simeq 3 \times 10^{15} \mathrm{~s}^{-1}$ at $300 \mathrm{~K}$ (typical of negative cation vacancies in compound semiconductors) and taking into account the $T^{-1 / 2}$ behavior to scale the coefficient at $400 \mathrm{~K}$, we estimate the $\mathrm{Ga}$ vacancy concentration from the value of $\tau_{\text {ave }}^{*}=214 \mathrm{ps}$ at $400 \mathrm{~K}$ as $\left[V_{\mathrm{Ga}}\right]=\left(\kappa_{V, \mathrm{Ga}} / \mu_{V, \mathrm{Ga}}\right) N_{\mathrm{at}} \simeq 6$ $\times 10^{17} \mathrm{~cm}^{-3}$, where $N_{\mathrm{at}}=8.9 \times 10^{22} \mathrm{~cm}^{-3}$ is the atomic density of $\mathrm{GaN}$. The irradiation fluence was $\phi(2 \mathrm{MeV})=5$ $\times 10^{17} \mathrm{~cm}^{-2}$, which gives an introduction rate $\Sigma_{\text {Ga }}^{2.0}$ $=\left[V_{\mathrm{Ga}}\right] / \phi \simeq 1.2 \mathrm{~cm}^{-1}$.

In addition to $\mathrm{Ga}$ vacancies, negative-ion-type defects are also produced in the irradiation. Their concentration can be estimated from their positron trapping rate at $200 \mathrm{~K}$, where the positrons no longer escape from the hydrogenlike Rydberg states around the negative ions. Keeping in mind the 5 ps adjustment, the trapping rate to the Ga vacancies at $400 \mathrm{~K}$ can be estimated as

$$
\kappa_{V, \mathrm{Ga}}=\lambda_{B} \frac{\tau_{\mathrm{ave}}^{*}-\tau_{B}}{\tau_{V, \mathrm{Ga}}-\tau_{\mathrm{ave}}^{*}} \simeq 1.6 \times 10^{10} \mathrm{~s}^{-1} .
$$

At $200 \mathrm{~K}$, where the positron escape from the Rydberg states is negligible, the trapping rate to the negative ions can be estimated (for more details on the procedure, see, e.g., Ref. 24) as

$$
\kappa_{\text {ion } 1}=\kappa_{V, \mathrm{Ga}} \frac{\tau_{V, \mathrm{Ga}}-\tau_{\mathrm{ave}}}{\tau_{\mathrm{ave}}-\tau_{B}} \simeq 9 \times 10^{10} \mathrm{~s}^{-1} .
$$

Here, we have used the $T^{-1 / 2}$ dependence of the trapping coefficient of the $\mathrm{Ga}$ vacancies, which gives $\kappa_{V, \mathrm{Ga}} \simeq 2.3$ $\times 10^{10} s^{-1}$ at $200 \mathrm{~K}$. The positron trapping coefficient to negative-ion-type defects is similar to that to negatively charged vacancies. Hence, the trapping rates estimated above indicate that the concentration of the negative-ion-type defects is four times that of the $\mathrm{Ga}$ vacancies, i.e., $c_{\text {ion } 1} \simeq 2.4$ $\times 10^{18} \mathrm{~cm}^{-3}$. Assuming that the $\mathrm{Ga}$ vacancies are produced
TABLE I. The experimentally obtained introduction rates (absolute accuracy within a factor of 2-3) of primary defects on both Ga and $\mathrm{N}$ sublattices compared to those predicted by theory. The theoretical introduction rates are calculated with the relativistic McKinley-Feshbach formula using both the minimum thresholds and the average thresholds averaged over a $15^{\circ}$ acceptance angle.

\begin{tabular}{lcc}
\hline \hline Method & $\begin{array}{r}\mathrm{Ga} \text { sublattice } \\
\left(E_{e}=2 \mathrm{MeV}\right)\end{array}$ & $\begin{array}{c}\mathrm{N} \text { sublattice } \\
\left(E_{e}=0.45 \mathrm{MeV}\right)\end{array}$ \\
\hline $\begin{array}{l}\text { Experiment } \\
\text { Theory, min. thresholds: }\end{array}$ & $5 \mathrm{~cm}^{-1}$ & $0.3 \mathrm{~cm}^{-1}$ \\
$\begin{array}{l}\text { Ga: } 22 \mathrm{eV}, \mathrm{N}: 25 \mathrm{eV} \\
\text { Theory, } 15^{\circ} \text { ave. thresholds: }\end{array}$ & $3 \mathrm{~cm}^{-1}$ & $0.6 \mathrm{~cm}^{-1}$ \\
Ga: $38 \mathrm{eV}, \mathrm{N}: 66 \mathrm{eV}$ & $1.4 \mathrm{~cm}^{-1}$ & $0.07 \mathrm{~cm}^{-1}$ \\
\hline \hline
\end{tabular}

together with the negative ions, we estimate the total Ga sublattice defect introduction rate directly from the negative ion concentration as $\Sigma_{\mathrm{Ga}}^{2.0}=c_{\text {ion1 }} / \phi \simeq 5 \mathrm{~cm}^{-1}$.

\section{Comparison of introduction rates with theory}

The theoretical minimum displacement energies $\left(E_{d}\right)$ are $25 \mathrm{eV}$ for the $\mathrm{N}$ displacement and $22 \mathrm{eV}$ for the Ga displacement, depending on the irradiation direction. ${ }^{25}$ However, along the $[000-1]$ direction as used here, and averaging over a $15^{\circ}$ acceptance angle to account for thermal motions and possible beam misalignment, the calculated displacement energies are 66 and $38 \mathrm{eV}$, respectively, for $\mathrm{N}$ and $\mathrm{Ga}$ displacements. ${ }^{2}$ The primary defect production rates can be calculated from these values with the McKinley-Feshbach relativistic displacement cross-section formula. ${ }^{1}$ The results calculated with both the minimum and $15^{\circ}$ averaged displacement energies are presented together with the ones obtained experimentally in this work in Table I.

As seen in the table, the experimental results for the $\mathrm{Ga}$ sublattice values are of the same order of magnitude and hence in reasonable agreement with the theoretical values, obtained with electron energy of $2 \mathrm{MeV}$. Further, the use of the minimum or $15^{\circ}$ averaged threshold energies has only little effect (a factor of 2) on the defect production rate because of the electron energy much higher than the minimum electron energy [0.53 MeV (Ref. 2)] needed for Ga displacement. The theoretical defect production rate on the $\mathrm{N}$ sublattice when using $2 \mathrm{MeV}$ electrons is a factor of 5-6 lower (not shown in the table) than that on the Ga sublattice. Hence, as seen in the experiments, the effect of the neutral $\mathrm{N}$ vacancies on the data in the $2 \mathrm{MeV}$ irradiated samples is negligible as the trapping rate should be an order of magnitude lower, and the dominant contribution to the negative ions originates from the damage in the Ga sublattice. Interestingly, the production rates calculated for the $\mathrm{N}$ sublattice with $0.45 \mathrm{MeV}$ electrons strongly depend on the threshold displacement energy used, the difference being one order of magnitude, due to the proximity of the minimum electron energy [0.32 MeV (Ref. 2)] needed for $\mathrm{N}$ displacement. The experimentally determined value lies between the two theoretical values. 
It should be noted that determining the value of the vacancy-specific trapping coefficient $\mu_{V}$ is rather difficult and one should consider the possibility of the coefficient differing even by a factor of 2-3 from the physically proper value. However, typical reported values of the trapping coefficient range from $0.5 \times 10^{15}$ to $5 \times 10^{15} \mathrm{~s}^{-1}$ at room temperature, the lower end for neutral and higher end for negatively charged vacancies. ${ }^{26}$ We have used in this work values $\mu_{V, \mathrm{~N}} \simeq 1 \times 10^{15} \mathrm{~s}^{-1}$ and $\mu_{V, \mathrm{Ga}} \simeq 3 \times 10^{15} \mathrm{~s}^{-1}$ for the neutral $\mathrm{N}$ and negative Ga vacancies, respectively. It is thus unlikely that the estimated vacancy concentrations and introduction rates were off by more than a factor of 2 .

Interestingly, for both sublattices and electron energies, the experimentally determined introduction rates are closer to those theoretical values where the minimum threshold energies (i.e., the smaller values) are used in the calculations. This suggests that the $15^{\circ}$ acceptance angle overestimates the spread of the incident electrons. Another possibility is that the molecular dynamics simulations used to estimate the theoretical threshold energies for atomic displacements give values that are too high.

\section{B. Recovery of the irradiation damage}

\section{Negative ions with different charge states}

As mentioned in Sec. III, the different temperatures at which the average positron lifetime starts increasing with temperature in the as-irradiated samples and samples annealed at $625 \mathrm{~K}$ and above indicate that the positron binding energies to the respective negative ions, causing the lifetime to be close to that of the bulk at low temperatures, are different. A rough estimate of the difference of these hydrogenic binding energies can be made by comparing the temperatures where the effects of the two negative ions are similar, indicating similar escape rates [see Eq. (1)]. In Fig. 4, it can be seen that roughly half of the effect of the negative ions with higher (ion 1) and lower (ion 2) binding energies occurs at about $T_{1}=350 \mathrm{~K}$ and $T_{2}=120 \mathrm{~K}$, respectively. Assuming that the two escape rates are equal at these temperatures, we obtain from Eq. (1)] a relation between the two binding energies: $E_{b \text {, ion } 1}=\left(T_{1} / T_{2}\right) E_{b, \text { ion } 2}+1.5 k T_{1} \ln \left(T_{1} / T_{2}\right) \simeq 3 E_{b \text {, ion } 2}$ $+35 \mathrm{meV}$. Hence, we obtain a lower limit for the ratio of the two binding energies as $E_{b \text {,ion1 } 1} / E_{b \text {,ion } 2}>3$.

Fitting the temperature dependent trapping model to the average lifetime vs temperature data gives the same trapping rates as those estimated directly in the previous section. Thus, no revisions are needed to the defect concentrations reported above. The binding energies of the negative ion defects are also obtained from the fit shown in Fig. 4 as $E_{b \text {,ion } 1}=100 \pm 20 \mathrm{meV}$ and $E_{b \text {,ion } 2}=25 \pm 10 \mathrm{meV}$. The ratio of these fitted binding energies is $E_{b \text {,ion } 1} / E_{b \text {,ion } 2}=4$, in excellent agreement with the rough estimate $(>3)$. As the binding energies are proportional to the square of the charge state, this result implies that the charge states of the two defects are different by a factor of 2 . Finally, as it is not likely for a defect to exhibit a charge state of an absolute value larger than 3, we conclude that the charge states of these two negative-ion-type defects observed in the irradiated samples are $2-$ and $1-$.
The average positron lifetime is constant as a function of temperature in the as-irradiated samples below $200 \mathrm{~K}$, with no further decrease below $100 \mathrm{~K}$. In addition, the average lifetime measured below $100 \mathrm{~K}$ does not increase in the annealings at temperatures $600-800 \mathrm{~K}$. These two observations indicate that the total concentration of the negative-iontype defects does not change in the annealings at those temperatures, where the negative ions with charge state 2disappear, and that the negative ions with charge state 1-are not present in the as-irradiated samples. We interpret this as the negative ions changing their charge state gradually, in two stages (as there is no evolution of the data at $625-700 \mathrm{~K}$ ), at annealing temperatures $600-800 \mathrm{~K}$.

Since both the $\mathrm{Ga}$ and $\mathrm{N}$ vacancies are observed as open volume defects by positrons, and the $\mathrm{N}$ sublattice damage is less important in $2 \mathrm{MeV}$ electron irradiation, we associate the negatively charged nonopen volume defects with $\mathrm{Ga}$ interstitials and/or antisites. Unfortunately, it is not possible to determine the identity of these negative defects with positron annihilation spectroscopy. However, as the isolated interstitials have been shown to be mobile already at room temperature, ${ }^{6,7}$ it is likely that the observed defects are stabilized by other defects or impurities. The complex annealing behavior supports this interpretation. As the concentrations of the other impurities are much below the concentrations of the negative ion defects, hydrogen is a prospective candidate for acting as a negative ion trap. Indeed, recovery temperatures in the range 700-900 $\mathrm{K}$ have been observed for ODEPR signals assigned to trapped $\mathrm{Ga}$ interstitials. ${ }^{6}$ We would like to take the opportunity here to encourage theorists to tackle the interactions of hydrogen with $\mathrm{Ga}$ interstitials and/or antisites in $\mathrm{GaN}$, as these defecthydrogen complexes are potentially important in determining the electrical properties and the annealing behavior of ion implantation processed $\mathrm{GaN}$.

\section{Defect dynamics in thermal annealings}

It was shown above that the trapping rates to the Ga vacancies and the negative-ion-type defects can be directly estimated from the positron data without fitting. Using Eq. (5), the trapping rates to the three defects in the $2 \mathrm{MeV}$ electronirradiated samples were calculated as a function of annealing temperature. They are presented in Fig. 6 together with the trapping rate to the $\mathrm{N}$ vacancies observed in the $0.45 \mathrm{MeV}$ electron-irradiated samples. The trapping rates are proportional to defect concentrations, but it should be noticed that they are estimated at different temperatures and hence do not represent the relations of the concentrations of the different defects: only the behavior as a function of annealing temperature of each defect separately is important here. We see in Fig. 6 that the Ga vacancies anneal out of the samples at two separate stages at around $600 \mathrm{~K}$ and above $1000 \mathrm{~K}$, the 2 - negative ions also anneal out at two separate stages at around $600 \mathrm{~K}$ (together with the first part of the Ga vacancies) and $700 \mathrm{~K}$, and the 1 - negative ions anneal out together with the second part of the Ga vacancies above $1000 \mathrm{~K}$. The $\mathrm{N}$ vacancies also anneal out around $600 \mathrm{~K}$.

The activation energies $E_{A}$ corresponding to the different annealing stages can be fitted to the positron data. Assuming 


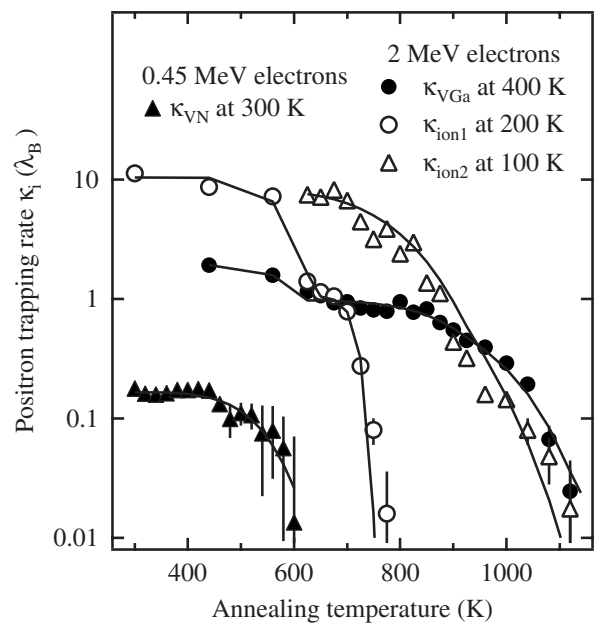

FIG. 6. The positron trapping rates to the different defects as a function of the annealing temperature $\left(\lambda_{B}=6.25 \times 10^{9} \mathrm{~s}^{-1}\right)$. The solid lines are fits to the data.

that each defect anneals independently, the isochronal annealing process for a defect concentration $N$ can be described $\operatorname{as}^{27}$

$$
N_{i+1}=N_{\infty}+\left(N_{i}-N_{\infty}\right) \exp \left[-\nu t \exp \left(-E_{A} / k T_{i}\right)\right]
$$

where the subscript $i$ denotes the annealing step $\left(T_{i}=[300\right.$ $+10(i-1)] \mathrm{K}), t=1800 \mathrm{~s}$ is the annealing time, and $\nu$ is a frequency factor (assumed to be $\nu=10^{13} \mathrm{~s}^{-1}$ ). The trapping rates are directly proportional to the defect concentrations. Thus, the trapping rates and activation energies of the annealing stages can be fitted to the data in Fig. 6 .

Using the above model, two activation energies for the 2negative ions were fitted, giving $E_{A 1}^{\text {ion1 }}=1.9 \pm 0.1 \mathrm{eV}$ and $E_{A 2}^{\mathrm{ion1}}=2.3 \pm 0.1 \mathrm{eV}$ (the error estimates are based on the variance of the fit). $90 \%$ of these negative ions disappear at the first annealing stage. The activation energy of the first annealing stage of the $\mathrm{Ga}$ vacancies is fitted as $E_{A 1}^{V, \mathrm{Ga}}$ $=1.8 \pm 0.1 \mathrm{eV}$, which naturally coincides with the first stage of the 2- negative ions. 50\% of the Ga vacancies anneal out of the samples at this first annealing stage. The activation energy of the annealing of the $\mathrm{N}$ vacancies is $E_{A}^{V, \mathrm{~N}}$ $=1.9 \pm 0.1 \mathrm{eV}$ as well.

The recovery of the 1- negative ions and the second stage of the Ga vacancies could not be fitted with a single activation energy. Instead, a linear dependence $E_{A}^{\mathrm{st}}=E_{A 0}^{\mathrm{st}}+\alpha\left(T_{i}\right.$ $-T_{1}$ ) was assumed. The fitting gave activation energies ranging from $2.6 \pm 0.1 \mathrm{eV}$ at the beginning of the anneal at about $800 \mathrm{~K}$ to $3.4 \pm 0.1 \mathrm{eV}$ at the end at about $1100 \mathrm{~K}$. This kind of "sliding" of the activation energy can be expected if the defects are not all identical and have different diffusion barriers, leading to a complex annealing behavior. Another possibility would be that the out-annealing of these defects is not limited only by diffusion, but e.g., by the transition to the surface or to another lattice site.

The first annealing stage of the Ga vacancies around $600 \mathrm{~K}$ is in excellent agreement with earlier experiments, ${ }^{8}$ where it was associated with the isolated Ga vacancy becom- ing mobile at this temperature, based on comparisons with ODEPR measurements. ${ }^{4-6}$ The migration barrier predicted by theoretical calculations for the 3- charge state of the isolated Ga vacancy is $1.9 \mathrm{eV} .{ }^{28}$ As the experimental activation energy coincides with this value, we obtain the migration barrier of the isolated $\mathrm{Ga}$ vacancy as $E_{M}^{V, \mathrm{Ga}}=E_{A 1}^{V, \mathrm{Ga}}$ $=1.8 \pm 0.1 \mathrm{eV}$.

The activation energies of 1.9 and $2.3 \mathrm{eV}$ of the changes in the charge state of the negative ions are likely to be associated with two subsequent reconstructions of the defects themselves. This interpretation is based on the general behavior of the Fermi level in the samples: in originally $n$-type material, the Fermi level typically moves toward the conduction band when the temperature is decreased, and when compensating irradiation-induced defects are removed by annealing. Hence, if the negative ion defects changed their charge state due to the movement of the Fermi level, they would become more negative in both scenarios, whereas in our experiments, they become less negative (from 2- to 1-). One possible explanation could be, e.g., the release of hydrogen from other defect complexes and the subsequent migration and trapping at these negative ions, making them less negative. However, as the structure of these defects remains unresolved, further experiments are needed in order to understand the observed behavior of the negative ions.

The fact that no single activation energy could be fitted to the annealing data above $800 \mathrm{~K}$, where the 1 - negative ions anneal out together with the other half of the Ga vacancies, indicates that complex migration processes are involved. As the isolated $\mathrm{Ga}$ vacancies are concluded to be mobile already at $600 \mathrm{~K}$, the remaining $\mathrm{Ga}$ vacancies need to be stabilized by other defects. $\mathrm{N}$ vacancies that are also produced in the $2 \mathrm{MeV}$ electron irradiation, although at a lower rate, and hydrogen are possible candidates as it would be difficult to distinguish Ga vacancy complexes with either (or both) of them from the isolated Ga vacancies. ${ }^{20}$ In addition, a significant part of the Ga vacancies cannot be complexed with oxygen as the $\mathrm{O}$ concentration is too low in our samples, and the breakup of $V_{\mathrm{Ga}}-\mathrm{O}_{\mathrm{N}}$ complexes has been shown to occur only above $1500 \mathrm{~K}^{.29-31}$ Interestingly, recent results of ODEPR measurements $^{7}$ (in high-purity HVPE GaN samples irradiated with $2.5 \mathrm{MeV}$ electrons to a similar fluence as in our work) were interpreted as isolated Ga vacancies surviving the annealing at $600 \mathrm{~K}$ and disappearing above $800 \mathrm{~K}$. However, the effect of hydrogen and Ga interstitials, which are likely to be positively charged and thus invisible to positrons, should be considered in more detail, and further experiments are needed here as well to clarify the situation (note that the $\mathrm{H}$ and $\mathrm{Ga}$ interstitials will be partly charged and partly neutral, since the Fermi level is likely to be close to their energy levels).

The predicted ${ }^{28}$ migration barriers for the $1+$ and $3+$ charge states of the $\mathrm{N}$ vacancy are 4.3 and $2.6 \mathrm{eV}$, respectively, both clearly higher than the activation energy observed here for the neutral $\mathrm{N}$ vacancy complex: $E_{A}^{V, \mathrm{~N}}$ $=1.8 \pm 0.1 \mathrm{eV}$. Interestingly, the same work predicts a migration barrier for the 1- charge state of the $\mathrm{N}$ interstitial (the samples are clearly $n$-type after the $0.45 \mathrm{MeV}$ electron irradiation) as $1.6 \mathrm{eV}$, slightly lower than the experimental activation energy. The $\mathrm{N}$ interstitials are likely to be the acceptor 
defects observed in Ref. 2. However, the concentration of these acceptors is much smaller than that of the neutral $\mathrm{N}$ vacancy complexes observed in our work. We propose that a significant part of the $\mathrm{N}$ interstitials that are produced together with the $\mathrm{N}$ vacancies also are complexed with hydrogen, producing neutral complexes invisible to both electrical and positron experiments. The presence of hydrogen in the complex does not necessarily increase significantly the migration barrier of the $\mathrm{N}$ interstitial, as (1) the predicted binding energy of such a complex is below $0.5 \mathrm{eV}$ in $n$-type $\mathrm{GaN}$ (Ref. 32) and (2) the complex could migrate without dissociation. Hence, we propose that the observed recovery of the $\mathrm{N}$ vacancies at $600 \mathrm{~K}$ is due to the $\mathrm{N}$ interstitials migrating and filling the vacancies.

\section{CONCLUSIONS}

We have applied positron annihilation spectroscopy to study the point defects introduced by 0.45 and $2 \mathrm{MeV}$ electron irradiation at room temperature in $n$-type GaN. The measurements of the as-irradiated samples were performed at $20-300 \mathrm{~K}$. The thermal recovery of the defects produced by the irradiation was studied by $30 \mathrm{~min}$ annealing of the samples at temperatures up to $1220 \mathrm{~K}$. The measurements were performed at room temperature, and the measurement temperature was scanned from 20 to $500 \mathrm{~K}$ after selected annealing steps.

As expected, we observe no Ga vacancies in the samples irradiated with $0.45 \mathrm{MeV}$ electrons. We do, however, observe smaller vacancy defects with specific lifetime of $\tau_{V}$ $=190 \pm 15$ ps that we tentatively identify as $\mathrm{N}$ vacancies or related complexes in the neutral charge state. The $\mathrm{N}$ vacancies are produced at a rate $\Sigma_{\mathrm{N}}^{0.45} \simeq 0.25 \mathrm{~cm}^{-1}$. The irradiation with $2 \mathrm{MeV}$ electrons produces negatively charged $\mathrm{Ga}$ vacancies and negative-ion-type defects (with no open volume) originating from the damage in the Ga sublattice, at a rate $\Sigma_{\mathrm{Ga}}^{2.0} \simeq 5 \mathrm{~cm}^{-1}$.
The irradiation-induced $\mathrm{N}$ vacancies anneal out of the samples at around $600 \mathrm{~K}$, possibly due to the motion of the irradiation-induced $\mathrm{N}$ interstitials, as the $\mathrm{N}$ vacancies themselves are predicted ${ }^{28}$ to be stable up to much higher temperatures. This in very good agreement with earlier experiments. ${ }^{1}$ Half of the irradiation-induced $\mathrm{Ga}$ vacancies anneal out of the samples also around $600 \mathrm{~K}$, and this is interpreted as the isolated $\mathrm{Ga}$ vacancies becoming mobile with a migration barrier of $E_{M}^{V, \mathrm{Ga}}=1.8 \pm 0.1 \mathrm{eV}$, in excellent agreement with both theory and earlier experiments. ${ }^{8,28}$ Interestingly, we observe a change of charge state of the irradiation-induced negative ions from $2-$ to $1-$ due to a reconstruction of the defects in two stages at annealing temperatures of about 600 and $700 \mathrm{~K}$. The negative ions anneal out of the samples together with the other half of the $\mathrm{Ga}$ vacancies (stabilized by, e.g., $\mathrm{N}$ vacancies and/or hydrogen) in thermal annealings at $800-1100 \mathrm{~K}$. No single activation energy could be fitted to the annealing behavior at these high temperatures, indicating that the recovery of these defects is not limited only by the diffusion in the lattice.

In summary, we have identified the vacancy defects introduced on both $\mathrm{Ga}$ and $\mathrm{N}$ sublattices in the 0.45 and $2 \mathrm{MeV}$ electron irradiation of high-purity HVPE GaN samples. The thermal annealings reveal the coexistence of different charge states of negative nonopen volume defects in the samples and that a significant part of the $\mathrm{Ga}$ vacancies introduced in the $2 \mathrm{MeV}$ electron irradiation is stabilized by other defects, making them survive annealing temperatures up to $1100 \mathrm{~K}$. All the irradiation-induced defects detectable by positrons are annealed out of the samples at $600 \mathrm{~K}$ in the case of the $0.45 \mathrm{MeV}$ electron irradiation and at $1120 \mathrm{~K}$ in the case of the $2 \mathrm{MeV}$ electron irradiation.

\section{ACKNOWLEDGMENTS}

We are grateful for the inspiring discussions with the late K. Saarinen. D.C.L. was supported by U.S. Air Force Contract No. FA8650-06-D-5401. *filip.tuomisto@tkk.fi

${ }^{1}$ D. C. Look, D. C. Reynolds, J. W. Hemsky, J. R. Sizelove, R. L. Jones, and R. J. Molnar, Phys. Rev. Lett. 79, 2273 (1997).

${ }^{2}$ D. C. Look, G. C. Farlow, P. J. Drevinsky, D. F. Bliss, and J. R. Sizelove, Appl. Phys. Lett. 83, 3525 (2003).

${ }^{3}$ Q. Yang, H. Feick, and E. R. Weber, Appl. Phys. Lett. 82, 3002 (2003).

${ }^{4}$ M. Linde, S. J. Uftring, G. D. Watkins, V. Härle, and F. Scholz, Phys. Rev. B 55, R10177 (1997).

${ }^{5}$ C. Bozdog, H. Przybylinska, G. D. Watkins, V. Härle, F. Scholz, M. Mayer, M. Kamp, R. J. Molnar, A. E. Wickenden, D. D. Koleske, and R. K. Henry, Phys. Rev. B 59, 12479 (1999).

${ }^{6}$ K. H. Chow, G. D. Watkins, A. Usui, and M. Mizuta, Phys. Rev. Lett. 85, 2761 (2000).

${ }^{7}$ K. H. Chow, L. S. Vlasenko, P. Johannesen, C. Bozdog, G. D. Watkins, A. Usui, H. Sunakawa, C. Sasaoka, and M. Mizuta, Phys. Rev. B 69, 045207 (2004).
${ }^{8}$ K. Saarinen, T. Suski, I. Grzegory, and D. C. Look, Phys. Rev. B 64, 233201 (2001).

${ }^{9}$ D. Gogova, A. Kasic, H. Larsson, B. Pécz, R. Yakimova, B. Magnusson, B. Monemar, F. Tuomisto, K. Saarinen, C. Miskys, M. Stutzmann, C. Bundesmann, and M. Schubert, Jpn. J. Appl. Phys., Part 1 43, 1264 (2004).

${ }^{10}$ D. Gogova, A. Kasic, H. Larsson, B. Monemar, F. Tuomisto, K. Saarinen, L. Dobos, B. Pécz, P. Gibart, and B. Beaumont, J. Appl. Phys. 96, 799 (2004).

${ }^{11}$ V. Darakchieva, T. Paskova, P. P. Paskov, B. Monemar, N. Ashkenov, and M. Schubert, J. Appl. Phys. 97, 013517 (2005).

${ }^{12}$ J. Karpinski, J. Jun, and S. Porowski, J. Cryst. Growth 66, 1 (1984).

${ }^{13}$ K. Saarinen, P. Hautojärvi, and C. Corbel, in Identification of Defects in Semiconductors, edited by M. Stavola (Academic, New York, 1998), p. 209.

${ }^{14}$ K. Saarinen, A. P. Seitsonen, P. Hautojärvi, and C. Corbel, Phys. 
Rev. B 52, 10932 (1994).

${ }^{15}$ C. Corbel, F. Pierre, K. Saarinen, P. Hautojärvi, and P. Moser, Phys. Rev. B 45, 3386 (1992).

${ }^{16}$ M. J. Puska, C. Corbel, and R. M. Nieminen, Phys. Rev. B 41, 9980 (1990).

${ }^{17}$ J. Oila, J. Kivioja, V. Ranki, K. Saarinen, D. C. Look, R. J. Molnar, S. S. Park, S. K. Lee, and J. Y. Han, Appl. Phys. Lett. 82, 3433 (2003).

${ }^{18}$ K. Saarinen, J. Nissilä, P. Hautojärvi, J. Likonen, T. Suski, I. Grzegory, B. Lucznik, and S. Porowski, Appl. Phys. Lett. 75, 2441 (1999).

${ }^{19}$ F. Tuomisto, K. Saarinen, B. Lucznik, I. Grzegory, H. Teisseyre, T. Suski, S. Porowski, P. Hageman, and J. Likonen, Appl. Phys. Lett. 86, 031915 (2005).

${ }^{20}$ S. Hautakangas, I. Makkonen, V. Ranki, M. J. Puska, K. Saarinen, X. Xu, and D. C. Look, Phys. Rev. B 73, 193301 (2006).

${ }^{21}$ S. Hautakangas, J. Oila, M. Alatalo, K. Saarinen, L. Liszkay, D. Seghier, and H. P. Gislason, Phys. Rev. Lett. 90, 137402 (2003).

${ }^{22}$ C. G. Van de Walle, Phys. Rev. B 56, R10020 (1997).

${ }^{23}$ B. Szucs, A. Gali, Z. Hajnal, P. Deak, and C. G. Van de Walle, Phys. Rev. B 68, 085202 (2003).

${ }^{24}$ F. Tuomisto, K. Saarinen, D. C. Look, and G. C. Farlow, Phys.
Rev. B 72, 085206 (2005).

${ }^{25}$ J. Nord, K. Nordlund, J. Keinonen, and K. Albe, Nucl. Instrum. Methods Phys. Res. B 202, 93 (2003).

${ }^{26}$ R. Krause-Rehberg and H. S. Leipner, Positron Annihilation in Semiconductors: Defect Studies, Springer Series in Solid-State Sciences (Springer-Verlag, Berlin, 1998).

${ }^{27}$ D. C. Look, D. C. Reynolds, J. W. Hemsky, R. L. Jones, and J. R. Sizelove, Appl. Phys. Lett. 75, 811 (1999).

${ }^{28}$ S. Limpijumnong and C. G. Van de Walle, Phys. Rev. B 69, 035207 (2004).

${ }^{29}$ F. Tuomisto, T. Suski, H. Teisseyre, M. Krysko, M. Leszczynski, B. Lucznik, I. Grzegory, S. Porowski, D. Wasik, A. Witowski, W. Gebicki, P. Hageman, and K. Saarinen, Phys. Status Solidi B 240, 289 (2003).

${ }^{30}$ T. Paskova, D. Hommel, P. P. Paskov, V. Darakchieva, B. Monemar, M. Bockowski, T. Suski, I. Grzegory, F. Tuomisto, K. Saarinen, N. Ashkenov, and M. Schubert, Appl. Phys. Lett. 88, 141909 (2006).

${ }^{31}$ F. Tuomisto, K. Saarinen, T. Paskova, B. Monemar, M. Bockowski, and T. Suski, J. Appl. Phys. 99, 066105 (2006).

${ }^{32}$ A. F. Wright, J. Appl. Phys. 90, 6526 (2001). 